\title{
Discrete structure of ultrathin dielectric films and their surface optical properties
}

\author{
S.V.Sukhov ${ }^{1}$ and K.V.Krutitsky ${ }^{1,2}$ \\ ${ }^{1}$ Ulyanovsk Branch of Moscow Institute of Radio Engineering \\ and Electronics of Russian Academy of Sciences, \\ P.B.9868, 48, Goncharov Str., Ulyanovsk 432011, Russia \\ e-mail: ufire@mv.ru \\ ${ }^{2}$ Fakultät für Physik, Universität Konstanz, Fach M 674, \\ D-78457 Konstanz, Germany \\ e-mail: kostya@spock.physik.uni-konstanz.de
}

October 25, 2018

\begin{abstract}
The boundary problem of linear classical optics about the interaction of electromagnetic radiation with a thin dielectric film has been solved under explicit consideration of its discrete structure. The main attention has been paid to the investigation of the near-zone optical response of dielectrics. The laws of reflection and refraction for discrete structures in the case of a regular atomic distribution are studied and the structure of evanescent harmonics induced by an external plane wave near the surface is investigated in details. It is shown by means of analytical and numerical calculations that due to the existence of the evanescent harmonics the laws of reflection and refraction at the distances from the surface less than two interatomic distances are principally different from the Fresnel laws. From the practical point of view the results of this work might be useful for the near-field optical microscopy of ultrahigh resolution.
\end{abstract}

PACS numbers: 78.20.-e, 81.40.Tv, 42.25.-p, 87.64.Xx

\section{Introduction}

Usually in the investigations of optical phenomena in dielectrics a medium is considered as a continuous system of radiators. Besides the macroscopic Maxwell equations are employed. This way of description works well if one considers the phenomena which take place at large enough distances from medium interfaces. However, in recent years the interest to the optical phenomena near the surfaces of different media at the distances much less than the radiation wavelength has dramatically increased. Beside a purely fundamental interest this is connected to the vigorous development of the scanning near-field optical microscopy [1]-[7]. There were developed various schemes of optical near-field microscopes and the work in this direction is still currently underway. However, all the schemes have a common feature, namely, the measurement 
of the optical response of the medium on the external radiation is carried out in the near zone at the distances of the order of surface inhomogenity [国, []].

The resolution obtained with the aid of near-field optical microscopes permanently increases and at the moment it is of the order of $10 \mathrm{~nm}$. Besides the probe scans the surface also at the distance of the order of $10 \mathrm{~nm}$ [7]. At such a small distance it becomes necessary to take into account the discrete structure of the medium. The attempts of consideration of discrete medium structure in the theoretical description of the near-field optical measurements have been undertaken in recent years [1]. However, the main attention has been paid to the situations when discrete dipoles model rather large surface inhomogenities and the spatial distribution of these dipoles is asymmetric. The progress in the near-field optical microscopy allows one to hope that there will be possible to observe the atomic structure of surfaces. Then it will be necessary to interpret the optical response from single atoms more or less regularly distributed on the surfaces. Similar problems occur when one wants to describe the near-field in the vicinity of the surfaces of photon [8] and plasmon [9] crystals. Besides it becomes more natural to employ microscopic equations.

Intensive studies on optics of discrete dielectric media have been started not very long time ago. The laws of refraction and reflection for discrete structures were shown to be different from Fresnel laws [10] and this leads to the anomalies in the behavior of the electromagnetic field near the surface [1]-[13]. Theorem of extinction for discrete structures has been derived and the corrections to the Snell law were worked out [14]. The characteristic features of the spontaneous emission process [15, 16], superradiation [17] and optical bistability [18] in discrete structures were investigated. The second harmonic generation in discrete structures has been considered in Ref. [19], where a good agreement with experimental data has been pointed out. It was also shown that the consideration of the discrete structure of the medium leads to the surface local-field effect [20] and surface induced optical anisotropy [21]-24].

Properties of the waves reflected from discrete structures in the wave and near zones have been studied as well. It has been shown that in the wave zone the reflected waves can be described by plane waves [10, 11, 25, 26, 27]. However, the treatment in Refs. [25, 26, 27] does not take into account evanescent harmonics which give a considerable contribution into optical response of discrete dielectric structures in the near zone. These evanescent harmonics are not related to the total internal reflection and they exist even in the case of a single atom [28]. Due to the neglect of the evanescent harmonics the method developed in Refs. [25, 26, 27] can be employed for the description of the optical response of a thick enough medium in the wave zone only.

A more general approach which implicitly takes into account the evanescent harmonics has been developed in Refs. [10, 11]. This allowed us to show that the fields of the reflected waves in the near zone are periodic functions of longitudinal coordinates with the period equal to the lattice constant. This result confirms the assumption put forward in Ref. [7] that with the aid of near-field optical microscopes one can observe the atomic structure of the medium surface. In the present paper we shall continue the investigations started in Refs. 10, 11]. Employing the methods of the calculation of the lattice sums developed in Refs. [12, 24 we are going to investigate in more details the behavior of evanescent harmonics near the surface of a dielectric film with an ideal crystal structure. We would like to note that the role of the evanescent harmonics in the near-zone optical response of dielectrics has been point out by Sivukhin 29]. On the basis of rather simple and intuitive physical arguments he came to the qualitative conclusion that the Fresnel laws of reflection and refraction have to be violated at the distances from the surface less than one interatomic distance. In the present paper we are 
going to undertake a quantitative analysis of this fundamental issue. Besides it will be shown that the existence of the evanescent harmonics leads to principal deviations from Fresnel laws of reflection and refraction at the distances from the surface of the order of few interatomic distances.

The paper is organized as follows. In section 2 we shall discuss the problem we are going to solve and the main equations which constitute the basis of the microscopic approach to the description of optical phenomena in the linear classical optics. Section 3 is devoted to the analyses of the probe influence on the electromagnetic field distribution in the film and the back influence of the perturbation caused by the probe on the field magnitude on it. It is shown that in some cases the probe influence can be neglected. In section 4 we shall calculate the distribution of the local field in a dielectric film under the incidence of a plane wave. In section 5 we shall analyze in details the characteristic features in the behavior of reflected and transmitted waves in the wave and near zones.

\section{Statement of the problem and basic equations}

Let a monochromatic light wave with frequency $\omega$ and electric field strength vector $\mathbf{E}_{I}(\mathbf{r}, t)=$ $\mathbf{A}_{I}(\mathbf{r}) \exp (-i \omega t)$ be incident on a dielectric film with thickness $h$. Above the film there is a probe which measures the electromagnetic field at a certain point of space. The probe will be treated as a single point-like dipole with a linear polarizability $\alpha_{p}$. Such a scheme can be realized in a real experiment using either a single atom in a magneto-optical trap [30] or an admixed atom implanted in the needle of a near-field microscope [7, 31]. Our aim is to investigate the behavior of the electromagnetic field in the film and to calculate the characteristics of the electromagnetic field on the probe that is moved (scanned) along the film surface at some fixed distance $d$. The field detection, which represents itself a separate problem, will not be touched in the present paper.

We shall treat the dielectric film as a system of $N$ monolayers located at a distance $a$ from one another. The atoms inside the monolayers possess a discrete spatial distribution. In a stationary case the equation for the local electric field strength on the probe $\mathbf{E}_{p}^{\prime}(\mathbf{r}, t)=\mathbf{E}_{p}(\mathbf{r}) \exp (-i \omega t)$ [32, 33] can be written down in the form

$$
\mathbf{E}_{p}(\mathbf{r})=\mathbf{A}_{I}(\mathbf{r})+i \frac{2}{3} k_{0}^{3} \alpha_{p} \mathbf{E}_{p}(\mathbf{r})+\alpha \sum_{j=1}^{N} \sum_{a_{j}} \nabla_{\mathbf{r}} \times \nabla_{\mathbf{r}} \times \mathbf{E}_{j}\left(\mathbf{r}_{a_{j}}^{\|}\right) G\left(R_{a_{j}}\right),
$$

where $\mathbf{r}=(x, y, z)$ is a radius-vector of the probe position; $\alpha$ is the microscopic polarizability of the atoms inside the film, which is assumed to be independent of the field. $G(R)=$ $\exp \left(i k_{0} R\right) / R$ is the Green function of Helmholtz equation, $k_{0}=\omega / c$ is the vacuum wave number, $\mathbf{R}_{a_{j}}=\mathbf{r}-\mathbf{r}_{a_{j}}, R_{a_{j}}=\left|\mathbf{R}_{a_{j}}\right|, \mathbf{r}_{a_{j}}=\left(\mathbf{r}_{a_{j}}^{\|}, z_{j}\right)$. We have also introduced the notation

$$
\mathbf{E}_{j}\left(\mathbf{r}_{a_{j}}^{\|}\right)=\mathbf{E}\left(\mathbf{r}_{a_{j}}^{\|}, z_{j}\right), \quad z_{j}=-(j-1) a, \quad j=\overline{1, N}
$$

The reference frame is chosen in such a manner that $z$ axis is perpendicular to the film surface. The second term on the r.h.s. of eq.(11) describes the Lorentz radiation damping [32, 34]. In spite of the fact that the radiation damping makes usually a small contribution into the resultant field the consideration of this term is of principal significance. If one does not take it into account the energy conservation is violated (see [35] and references therein). 
Let $\mathbf{r}_{a_{l}}=\left(x_{a_{l}}, y_{a_{l}}, z_{l}\right)$ be a radius-vector of some atom in the $l$-th monolayer. Then for the local field at the position of this atom we have

$$
\begin{aligned}
\mathbf{E}_{l}\left(\mathbf{r}_{a_{l}}^{\|}\right) & =\mathbf{E}_{I l}\left(\mathbf{r}_{a_{l}}^{\|}\right)+i \frac{2}{3} k_{0}^{3} \alpha \mathbf{E}_{l}\left(\mathbf{r}_{a_{l}}^{\|}\right)+\alpha_{p} \nabla_{\mathbf{r}_{a_{l}}} \times \nabla_{\mathbf{r}_{a_{l}}} \times \mathbf{E}_{p}(\mathbf{r}) G\left(R_{a_{l}}\right) \\
& +\alpha \sum_{j=1}^{N} \sum_{a_{j}} \nabla_{\mathbf{r}_{a_{l}}} \times \nabla_{\mathbf{r}_{a_{l}}} \times \mathbf{E}_{j}\left(\mathbf{r}_{a_{j}}^{\|}\right) G\left(R_{a_{l} a_{j}}\right), \quad l=\overline{1, N}
\end{aligned}
$$

where $\mathbf{E}_{I l}\left(\mathbf{r}_{a_{l}}^{\|}\right)=\mathbf{A}_{I}\left(\mathbf{r}_{a_{l}}\right), R_{a_{l} a_{j}}=\left|\mathbf{r}_{a_{l}}-\mathbf{r}_{a_{j}}\right|$. Note that the term with $\mathbf{r}_{a_{j}}=\mathbf{r}_{a_{l}}$ has to be excluded from the summation.

We can look for the solution in the $l$-th monolayer of the film in the form

$$
\mathbf{E}_{l}\left(\mathbf{r}_{a_{l}}^{\|}\right)=\mathbf{E}_{l}^{0}\left(\mathbf{r}_{a_{l}}^{\|}\right)+\mathbf{E}_{l}^{p}\left(\mathbf{r}_{a_{l}}^{\|}\right)
$$

and in addition we impose a requirement that the field $\mathbf{E}_{l}^{0}\left(\mathbf{r}_{a_{l}}^{\|}\right)$has to satisfy the equation

$$
\mathbf{E}_{l}^{0}\left(\mathbf{r}_{a_{l}}^{\|}\right)=\mathbf{E}_{I l}\left(\mathbf{r}_{a_{l}}^{\|}\right)+i \frac{2}{3} k_{0}^{3} \alpha \mathbf{E}_{l}^{0}\left(\mathbf{r}_{a_{l}}^{\|}\right)+\alpha \sum_{j=1}^{N} \sum_{a_{j}} \nabla_{\mathbf{r}_{a_{l}}} \times \nabla_{\mathbf{r}_{a_{l}}} \times \mathbf{E}_{j}^{0}\left(\mathbf{r}_{a_{j}}^{\|}\right) G\left(R_{a_{l} a_{j}}\right)
$$

Then from eqs.(1), (3) it follows that the fields $\mathbf{E}_{l}^{p}$ and $\mathbf{E}_{p}$ will be the solutions of the system of equations

$$
\begin{aligned}
\mathbf{E}_{p}(\mathbf{r})=\mathbf{A}_{I}(\mathbf{r})+i \frac{2}{3} k_{0}^{3} \alpha_{p} \mathbf{E}_{p}(\mathbf{r}) & +\alpha \sum_{j=1}^{N} \sum_{a_{j}} \nabla_{\mathbf{r}} \times \nabla_{\mathbf{r}} \times \mathbf{E}_{j}^{0}\left(\mathbf{r}_{a_{j}}^{\|}\right) G\left(R_{a_{j}}\right) \\
& +\alpha \sum_{j=1}^{N} \sum_{a_{j}} \nabla_{\mathbf{r}} \times \nabla_{\mathbf{r}} \times \mathbf{E}_{j}^{p}\left(\mathbf{r}_{a_{j}}^{\|}\right) G\left(R_{a_{j}}\right), \\
\mathbf{E}_{l}^{p}\left(\mathbf{r}_{a_{l}}^{\|}\right)=i \frac{2}{3} k_{0}^{3} \alpha \mathbf{E}_{l}^{p}\left(\mathbf{r}_{a_{l}}^{\|}\right) & +\alpha_{p} \nabla_{\mathbf{r}_{a_{l}}} \times \nabla_{\mathbf{r}_{a_{l}}} \times \mathbf{E}_{p}(\mathbf{r}) G\left(R_{a_{l}}\right) \\
& +\alpha \sum_{j=1}^{N} \sum_{a_{j}} \nabla_{\mathbf{r}_{a_{l}}} \times \nabla_{\mathbf{r}_{a_{l}}} \times \mathbf{E}_{j}^{p}\left(\mathbf{r}_{a_{j}}^{\|}\right) G\left(R_{a_{l} a_{j}}\right) .
\end{aligned}
$$

The physical meaning of the fields $\mathbf{E}_{l}^{0}$ and $\mathbf{E}_{l}^{p}$ is the following. $\mathbf{E}_{l}^{0}$ is the local field inside the film without consideration of the contribution made by the probe. This contribution is taken into account by means of the field $\mathbf{E}_{l}^{p}$. Thus, for the solution of the problem stated above it is necessary first to solve the unperturbed problem (5). Then using the obtained solution one can determine the perturbation of the field inside the film caused by the probe and calculate the back influence of this perturbation on the field at the position of the probe (6), (7). However, before dealing with this general problem we shall estimate the perturbation in the film caused by the probe and find out under which conditions it is considerable.

\section{Estimate of the perturbation caused by the probe}

In this section we shall estimate $\mathbf{E}_{l}^{p}$ which enters eqs.(6), (7). For that purpose one can neglect radiation damping terms in eqs.(6), (17). Our starting point is the following. The perturbation 
of the field in the medium $\mathbf{E}_{l}^{p}$, which is caused by the probe, decreases rapidly with the increase of the distance between the surface and the probe. If in addition we take into account that the influence of atoms on one another also rapidly decreases with the distance, one can come to the conclusion that either the probe or the nearby atoms will be influenced mainly by the atoms located in the nearest vicinity around them. Thus, it is necessary to find the field $\mathbf{E}^{p}\left(\mathbf{r}_{a}\right)$ in the direct vicinity of the probe. Let's estimate first the size of this region.

One can assume that the behavior of the field $\mathbf{E}_{l}^{p}$ has the same features as that induced by the probe

$$
\begin{aligned}
\mathbf{E}_{l}^{p}\left(\mathbf{r}_{a_{l}}^{\|}\right) \propto & {\left[\left(\frac{3\left(\mathbf{E}_{p} \mathbf{R}_{a_{l}}\right) \mathbf{R}_{a_{l}}}{R_{a_{l}}^{5}}-\frac{\mathbf{E}_{p}}{R_{a_{l}}^{3}}\right)-i k_{0}\left(\frac{3\left(\mathbf{E}_{p} \mathbf{R}_{a_{l}}\right) \mathbf{R}_{a_{l}}}{R_{a_{l}}^{4}}-\frac{\mathbf{E}_{p}}{R_{a_{l}}^{2}}\right)-\right.} \\
& \left.k_{0}^{2}\left(\frac{\left(\mathbf{E}_{p} \mathbf{R}_{a_{l}}\right) \mathbf{R}_{a_{l}}}{R_{a_{l}}^{3}}-\frac{\mathbf{E}_{p}}{R_{a_{l}}}\right)\right] \exp \left(i k_{0} R_{a_{l}}\right) .
\end{aligned}
$$

Taking into account that the field on the probe is defined by the atoms in the near zone, one can keep in eq.(8) only the terms proportional to $R_{a_{l}}^{-3}$. We substitute the corresponding expression into (7) and replace the summation by integration. As a result of this the expression for the third term in the r.h.s. of (]) takes the form

$$
\alpha \sum_{j=1}^{N} \sum_{a_{j}} \nabla_{\mathbf{r}_{a_{l}}} \times \nabla_{\mathbf{r}_{a_{l}}} \times \mathbf{E}_{j}^{p}\left(\mathbf{r}_{a_{j}}^{\|}\right) G\left(R_{a_{l} a_{j}}\right) \rightarrow \rho \int_{\Sigma_{\sigma}}^{\Sigma} \nabla_{\mathbf{r}} \times \nabla_{\mathbf{r}} \times \mathbf{E}^{p}\left(\mathbf{r}^{\prime}\right) d \mathbf{r}^{\prime}
$$

with $\rho$ being the macroscopic concentration of atoms. The integration in (9) is carried out over the film volume limited by the external surface $\Sigma$. In order to prevent the interaction of the atom with itself a small region limited by the spherical surface $\Sigma_{\sigma}$ centered at the observation point has to be excluded from the integration. The size $\sigma$ of this small region is of the order of lattice constant. In Ref. [10 it was shown that in order to achieve the complete correspondence passing from discrete to continuous atomic distribution one has to put $\sigma=\frac{3}{4} a$.

Let's determine the region of space, atoms from which make the main contribution to the field on the atom nearest to to the probe. Let $\delta$ be a tolerance of the calculations of the dipole field. Then

$$
1-\int_{\Sigma_{\sigma}}^{\Sigma_{L}} \nabla_{\mathbf{r}} \times \nabla_{\mathbf{r}} \times \mathbf{E}^{p}\left(\mathbf{r}^{\prime}\right) d \mathbf{r}^{\prime} / \int_{\Sigma_{\sigma}}^{\Sigma} \nabla_{\mathbf{r}} \times \nabla_{\mathbf{r}} \times \mathbf{E}^{p}\left(\mathbf{r}^{\prime}\right) d \mathbf{r}^{\prime}=\delta
$$

Integration in the denominator and in the numerator in eq.(10) is carried out over the film volume and over the spherical segment with the radius $L$ centered at the position of the atom nearest to the probe, respectively. Taking into account (8) we obtain from eq.(10) the following estimate for the radius of the sphere $L$ within which it is necessary to calculate the fields on the atoms:

$$
L \approx \sigma \delta^{-1 / 3}
$$

Let $\delta=0.1 \%$, then $L \approx 7 a$. Note that in the calculations one can use even less values of $L$, because in eq.(7) there is also the field induced by the probe and compared to this field the influence of the remote atoms is less. The conclusion that only the neighboring atoms contribute to the field at the site of probe in near-field regime is in agreement with the results of Ref. [36], where the influence on the probe caused by microscopic dielectric spheres located at the surface has been calculated. It was shown that the lattice of $5 \times 5$ spheres makes the main contribution. The increase of this area does not influence the final result. 
Performing analogous calculations for eq.(6) we obtain that the main contribution to the field on the probe is made by the atoms located inside the sphere with the radius $L$ centered at the probe position. Thus, the system of equations (6), ([7) is reduced to the system of few linear algebraic equations, which can be solved numerically after the field $\mathbf{E}_{l}^{0}$ is obtained from eq.(5). However, as it will be shown below in many situations it is even not necessary to solve this system of equations due to the negligible contribution of $\mathbf{E}^{p}$.

For the sake of distinctness we assume the probe to be located above some surface atom of the film. The field induced by the probe at the position of this atom is determined by the expression

$$
\mathbf{E}_{e f f} \approx-\alpha_{p} \mathbf{E}_{p} / d^{3},
$$

where $d$ is the distance between the probe and the atom. In formula (12) we have neglected the retardation and the field on the probe is chosen to be polarized parallel to the film surface assuming that the variations of the direction of the polarization vector does not influence much the final results. It has been shown above that in the calculations of the field on the probe it is enough to take into account the atoms located inside the sphere with the radius $L$. The rest of the film does not influence much the probe. So we shall estimate the influence on the probe, caused by the atoms located within the spherical segment centered at the position of the atom below the probe. Obviously, the microscopic field amplitude on the atom under consideration is larger than that on the other atoms of the film. The upper estimate of the field amplitude at the position of this atom $\mathbf{E}_{\max }$ is given by

$$
\left|\mathbf{E}_{\text {max }}\right| \leq\left|\mathbf{E}_{e f f}\right|[1-1.71 C \ln (L / \sigma)]^{-1}
$$

where the dimensionless parameter $C=\alpha \rho$ is a volumic polarizability [27]. We shall assume that the field on any atom inside the spherical segment is constant and equal to the field on the central atom $\mathbf{E}_{\max }$. It is obvious that in this approximation the influence on the probe caused by the spherical segment is larger than it is. Our calculations are approximate, so we replace the summation by the integration. Calculating the field at the position of the probe we get an upper estimate of the last term in the r.h.s. of eq.(6)

$$
\left|\mathbf{E}_{R}^{e f f}\right|<1.71 C\left|\mathbf{E}_{\max }\right| \ln (1+L / d) .
$$

Taking into account (12), (13), (14), we get a condition under which one can neglect this term:

$$
\frac{d^{3}}{\alpha_{p}} \gg \frac{1.71 C \ln (1+L / d)}{[1-1.71 C \ln (L / \sigma)]} .
$$

Note that the condition (15) does not depend on the way of excitation of the surface atoms. It remains the same if the atoms are excited by a wave incident from the side of the probe, by an evanescent wave, or by the wave radiated by the probe itself.

In eq.(15) $\ln (L / d+1)$ and $\ln (L / \sigma)$ are of the order of unity. Therefore, one can rewrite (15) in a simpler form

$$
\frac{d^{3}}{\alpha_{p}} \gg \frac{1.71 C}{1-1.71 C} .
$$

Usually for dielectric media $C \sim 0.1$ and $d^{3} / \alpha_{p} \sim 10$ at the distances from the surface equal to one lattice constant. Therefore, inequality (16) is satisfied at the distances equal or greater than one lattice constant. 
At the distances between the probe and the surface such that the influence of discrete atomic distribution is negligible but the retardation is still important the calculation of the field on the probe reduces to the electrostatic problem of the calculation of the dipole field near a dielectric surface. For the field $\mathbf{E}_{R}^{e f f}$ at the probe position we get

$$
\mathbf{E}_{R}^{e f f} \approx \frac{\pi}{4} \frac{C}{1+\frac{2}{3} \pi C} \frac{\alpha_{p}}{d^{3}}\left(\mathbf{E}_{p}-3\left(\mathbf{E}_{p} \mathbf{n}\right) \mathbf{n}\right)
$$

where $\mathbf{n}=(0,0,1)$ is a unit vector perpendicular to the monolayer. We write here " $\approx$ ", because in the derivation of eq.(17) we have used the Lorentz-Lorenz formula for the refractive index $n$ :

$$
n^{2}=\frac{1+(8 \pi / 3) C}{1-(4 \pi / 3) C}
$$

which does not describe properly optical properties in the surface region [37]. The condition which allows to neglect the last term in eq.(17) is given now by

$$
\frac{d^{3}}{\alpha_{p}} \gg \frac{\pi}{4} \frac{C}{1+\frac{2}{3} \pi C}
$$

The condition (19) is in a good agreement with (16), albeit the former imposes less strict limitations on $d$.

Thus, at the distances between the probe and the surface of the order of one lattice constant the back influence of the film on the probe can be safely neglected. In this approximation the field on the probe is determined by the field of the external wave and the field $\mathbf{E}_{l}^{0}$. The problem of calculation of the field $\mathbf{E}_{l}^{0}$ will be discussed in the next section.

\section{Distribution of the field inside the film (solution of the unperturbed problem)}

Let the external wave be a plane one, i.e.,

$$
\mathbf{A}_{I}(\mathbf{r})=\mathbf{E}_{0 I} \exp \left(i \mathbf{k}_{0} \mathbf{r}\right), \mathbf{k}_{0}=k_{0}\left(\sin \Theta_{I} \cos \Phi_{I}, \sin \Theta_{I} \sin \Phi_{I},-\cos \Theta_{I}\right),
$$

where $\Theta_{I}$ is an incident angle and the azimuthal angle $\Phi_{I}$ defines the orientation of the incidence plane.

The atoms of the monolayers are assumed to form a regular periodic structure with elementary translations vectors $\mathbf{a}_{1}$ and $\mathbf{a}_{2}$. Without loss of generality one can put

$$
\mathbf{a}_{1}=a_{\|}(1,0,0), \quad \mathbf{a}_{2}=a_{\|}(\alpha, \beta, 0) .
$$

For the sake of simplicity the vectors $\mathbf{a}_{1}$ and $\mathbf{a}_{2}$ are assumed to be the same for all monolayers. The generalization on different $\mathbf{a}_{1}$ and $\mathbf{a}_{2}$ for different monolayers can be easily done. In these notations the radius-vector of some atom in the $j$-th monolayer has the form

$$
\mathbf{r}_{a_{j}}=\mathbf{r}_{j}^{0}+\mathbf{a}_{m n}=\mathbf{r}_{j}^{0}+m \mathbf{a}_{1}+n \mathbf{a}_{2}
$$

where $m$ and $n$ are integer. The vector $\mathbf{r}_{j}^{0}$ has the following form

$$
\mathbf{r}_{j}^{0}=\alpha_{1 j} \mathbf{a}_{1}+\alpha_{2 j} \mathbf{a}_{2}+z_{j} \mathbf{n}, \quad 0 \leq \alpha_{1(2) j}<1 .
$$


Nonvanishing $\alpha_{1 j}$ and $\alpha_{2 j}$ take into account possible parallel shifts of the atomic planes relative to one another.

Making use of the principle of parallel translational symmetry the solution for the local field in the $l$-th monolayer can be written down in the form [12, 13, 20, 22, 24]:

$$
\mathbf{E}_{l}^{0}\left(\mathbf{r}^{\|}\right)=\mathbf{E}_{l}^{0} \exp \left(i \mathbf{k}_{0} \mathbf{r}^{\|}\right)
$$

We substitute (20), (24) into (5). Now it is necessary to calculate the lattice sums in eq.(5). Due to the slow convergence of lattice sums their direct calculation is a very laborious and in fact impracticable computational problem. In order to compute them one can use "the Lorentz method" according to which the atoms located near the observation point are treated as discretely distributed and the atoms outside a fictitious boundary are treated as continuously distributed. This method can be employed either for well-ordered or for random media and for the observation points either inside or outside of the media. This way of computation has been employed in various modifications in Refs. [10, 11, 20, 37, 38]. On the other hand, if the atomic distribution in the monolayers is regular, this separation of discrete and continuous regions is not necessary anymore. In this case there exist another way of computation of the lattice sums which is rapidly convergent and more convenient for the interpretation of the field behavior near the surface [12, 24]. It is this latter way will be employed in the present paper.

If the observation point is located not in the monolayer over which the summation is carried out $(j \neq l)$ the lattice sums can be converted to the form which is more appropriate for numerical calculations, making use of the formula for the dipole field induced by the $j$-th monolayer at some point $\mathbf{r}$, which has been obtained in Ref. [24] by means of Fourier transform (Ewald's three-fold integral transform):

$$
\begin{aligned}
& \mathbf{E}\left(\mathbf{r}, z_{j}\right)= \alpha \sum_{a_{j}} \nabla_{\mathbf{r}} \times \nabla_{\mathbf{r}} \times \mathbf{E}_{j}^{0} \exp \left(i \mathbf{k}_{0} \mathbf{r}_{a_{j}}^{\|}\right) G\left(R_{a_{j}}\right) \\
&= \sum_{p, q=-\infty}^{\infty} \mathbf{A}_{p q}\left(\mathbf{r}, z_{j}\right) \exp \left(i \mathbf{k}_{0}^{\|} \mathbf{r}\right), z \neq z_{j}, \\
& \mathbf{A}_{p q}\left(\mathbf{r}, z_{j}\right)=-\frac{2 \pi i \alpha}{\left|\mathbf{a}_{1} \times \mathbf{a}_{2}\right|}\left[\mathbf{k}_{p q} \times\left(\mathbf{k}_{p q} \times \mathbf{E}_{j}^{0}\right)\right] \frac{\exp \left[i\left(\mathbf{k}_{p q}-\mathbf{k}_{0}^{\|}\right)\left(\mathbf{r}-\mathbf{r}_{j}^{0}\right)\right]}{\kappa_{p q}}, \\
& \mathbf{k}_{p q}= \begin{cases}\left(\mathbf{k}_{0}^{\|}+\mathbf{g}_{p q}^{\|}, \kappa_{p q}\right) & \text { if } z>z_{j} \\
\left(\mathbf{k}_{0}^{\|}+\mathbf{g}_{p q}^{\|},-\kappa_{p q}\right) & \text { if } z<z_{j} ; \\
\kappa_{p q}=\sqrt{k_{0}^{2}-\left(\mathbf{k}_{0}^{\|}+\mathbf{g}_{p q}^{\|}\right)^{2}}, & \mathbf{g}_{p q}^{\|}=p \mathbf{g}_{1}+q \mathbf{g}_{2} \\
\mathbf{k}_{0}^{\|}= & k_{0}\left(\sin \Theta_{I} \cos \Phi_{I}, \sin \Theta_{I} \sin \Phi_{I}, 0\right) .\end{cases}
\end{aligned}
$$

The vectors of the reciprocal lattice $\mathbf{g}_{1}$ and $\mathbf{g}_{2}$ are related to the vectors $\mathbf{a}_{1}$ and $\mathbf{a}_{2}$ as

$$
\mathbf{g}_{1}=2 \pi \frac{\mathbf{a}_{2} \times \mathbf{n}}{\left|\mathbf{a}_{1} \times \mathbf{a}_{2}\right|}, \quad \mathbf{g}_{2}=2 \pi \frac{\mathbf{n} \times \mathbf{a}_{1}}{\left|\mathbf{a}_{1} \times \mathbf{a}_{2}\right|}
$$

The choice of the vectors $\mathbf{a}_{1}$ and $\mathbf{a}_{2}$ can be arbitrary. However, it makes sense to choose them in such a manner that they have a minimal length. Then the length of the vectors $\mathbf{g}_{1}$ and $\mathbf{g}_{2}$ will be also minimal. Such a choice of the basis is convenient, because in this case the 
decay coefficients of the evanescent waves for square lattices and for slightly anisotropic lattices increase with the parameter $\kappa=p^{2}+q^{2}$.

Formula (25) is a useful representation of the lattice sums for numerical calculations because the sums over $p, q$ converge much faster than the sums over $a_{j}$, provided that $\left|z-z_{j}\right| \geq a_{\|}$. The calculations carried out in the next section show that in the sum over $p, q$ it is enough to keep only few summands with minimal $g_{p q}^{\|}$.

On the other hand, formula (25) has a very clear physical interpretation. It represents itself a decomposition of the field induced by the monolayer of discrete atoms on propagating and evanescent waves. Indeed, if $\left|\mathbf{k}_{0}^{\|}+\mathbf{g}_{p q}^{\|}\right|<k_{0}$ the quantity $\kappa_{p q}$ is real and we have propagating waves. Otherwise, $\kappa_{p q}$ is imaginary and we have exponentially decaying waves. A more detailed discussion of the role of propagating and evanescent waves will be given in the next section.

Note that the quantities $\mathbf{A}_{p q}\left(\mathbf{r}, z_{j}\right)$ with $p, q \neq 0$, i.e. the evanescent harmonics, are periodic functions of $x$ and $y$ and they posses the following property of the translational symmetry

$$
\mathbf{A}_{p q}\left(\mathbf{r}+m \mathbf{a}_{1}+n \mathbf{a}_{2}, z_{j}\right)=\mathbf{A}_{p q}\left(\mathbf{r}, z_{j}\right) .
$$

As we shall see further on, this property of the evanescent harmonics leads to the fact that the intensities of the reflected and transmitted waves near the film surface are periodic functions of $x$ and $y$.

If the observation point $\mathbf{r}$ is at the position of some atom inside the monolayer over which the summation is carried out $(j=l)$, then for the calculation of the lattice sum it is convenient to employ the method based on Ewald's onefold integral transform [12]. Separating the initial sum over the real lattice on the sums over the real and reciprocal lattices one gets the following expression for the dipole field [12]

$$
\alpha \sum_{a_{j}} \nabla_{\mathbf{r}} \times \nabla_{\mathbf{r}} \times\left.\mathbf{E}_{j}^{0} \exp \left(i \mathbf{k}_{0} \mathbf{r}_{a_{j}}^{\|}\right) G\left(R_{a_{j}}\right)\right|_{z=z_{j}}+i \frac{2}{3} k_{0}^{3} \alpha \mathbf{E}_{j}^{0} \exp \left(i \mathbf{k}_{0}^{\|} \mathbf{r}\right)=\alpha \hat{f}\left(\mathbf{k}_{0}\right) \mathbf{E}_{j}^{0} \exp \left(i \mathbf{k}_{0}^{\|} \mathbf{r}\right)
$$

where the term with $\mathbf{r}^{\|}=\mathbf{r}_{a_{j}}^{\|}$has to be excluded from the summation. Tensor $\hat{f}$ is a symmetric one and the components $f_{x z}, f_{y z}, f_{z x}, f_{z y}$ vanish. It has the form [12

$$
\begin{aligned}
f^{\nu \mu}(\mathbf{k})= & c^{\nu \mu}-\left[\frac{2}{3} i k^{3} \operatorname{erfc}\left(\frac{i k}{2 E}\right)+\frac{4 E}{3 \sqrt{\pi}}\left(k^{2}-E^{2}\right) \exp \left(\frac{k^{2}}{4 E^{2}}\right)-\frac{2}{3} i k^{3}\right] \delta_{\nu \mu} \\
c^{\nu \mu}= & \frac{i \pi}{\left|\mathbf{a}_{1} \times \mathbf{a}_{2}\right|} \sum_{p, q}\left[\frac{k^{2} \delta_{\nu \mu}-k_{p q \nu} k_{p q \mu}}{k_{p q}} \Delta_{p q}(1+\tau)+\eta \Sigma_{p q}\right] \\
& +\frac{1}{2} \sum_{n, m} \frac{\exp \left(i \mathbf{k} \mathbf{a}_{n m}\right)}{a_{n m}^{3}}\left\{\Gamma_{n m}^{1}\left[\delta_{\nu \mu} \Gamma_{n m}^{2}+\mathbf{a}_{n m}^{\nu} \mathbf{a}_{n m}^{\mu} \Gamma_{n m}^{3}\right]\right. \\
& \left.+\Gamma_{n m}^{4}\left[-\delta_{\nu \mu} a_{n m}+\mathbf{a}_{n m}^{\nu} \mathbf{a}_{n m}^{\mu} \Gamma_{n m}^{5}\right]+\text { c.c. }\right\},
\end{aligned}
$$

where $\nu, \mu=x, y, z, a_{n m}=\left|\mathbf{a}_{n m}\right|$

$$
\begin{gathered}
\eta=\delta_{\mu z} \delta_{\nu z}, \quad \tau=(-1)^{\delta_{\mu z}}(-1)^{\delta_{\nu z}} \\
\Delta_{p q}=\operatorname{erfc}\left(-\frac{i k_{p q}}{2 E}\right) \quad, \quad \Sigma_{p q}=\frac{i 4 E}{\sqrt{\pi}} \exp \left(\frac{k_{p q}^{2}}{4 E^{2}}\right) \\
\Gamma_{n m}^{1}=\exp \left(-i k a_{n m}\right) \operatorname{erfc}\left(a_{n m} E-\frac{i k}{2 E}\right) \quad, \quad \Gamma_{n m}^{2}=-1-i k a_{n m}+k^{2} a_{n m}^{2}
\end{gathered}
$$




$$
\Gamma_{n m}^{3}=-k^{2}+\frac{3 i k}{a_{n m}}+\frac{3}{a_{n m}^{2}} \quad, \quad \Gamma_{n m}^{4}=\frac{2 E}{\sqrt{\pi}} \exp \left(-E^{2} a_{n m}^{2}+\frac{k^{2}}{4 E^{2}}\right) \quad, \quad \Gamma_{n m}^{5}=\frac{3}{a_{n m}}+2 E^{2} a_{n m} .
$$

The rate of convergence of the sums in (31) is determined by the parameter $E$, which has a dimension of the inverse length. It appears as a result of the formal separation of the initial sum over the real lattice on the sums over the real and reciprocal lattices. This parameter has no any physical meaning, it must be only real and positive and its choice can be arbitrary [12]. However, it makes sense to choose the parameter $E$ in such a manner that the rates of convergence for the sums over $(p, q)$ and $(m, n)$ are the same. One can show that this requirement is fulfilled, provided that $E=\sqrt{\pi /\left|\mathbf{a}_{1} \times \mathbf{a}_{2}\right|}$. Substituting this value into (31) one can estimate the maximal values of $(n, m)$ and $(p, q)$ which has to be taken for the calculation of the lattice sums with the tolerance $\varepsilon$. Taking into account that at large $x \operatorname{erfc}(x) \approx \exp \left(-x^{2}\right) /(x \sqrt{\pi})$, we get the condition

$$
\exp \left(-\pi a_{n m}^{2} /\left|\mathbf{a}_{1} \times \mathbf{a}_{2}\right|\right) \approx \varepsilon
$$

Exact numerical calculations for a square lattice show that if the summation is restricted by the terms with $n, m, p, q=0, \pm 1$ the relative tolerance of the calculations of the dipole field is about $10^{-6}$, which is in a good agreement with the estimate (32). Therefore, our choice of the parameter $E$ appears to be very convenient for carring out numerical calculations.

Note that in the computer calculations of the dipole field the algorithm based on Ewald's onefold integral transform is 5-10 times faster than that based on the Lorentz method.

Taking into account identities (25), (30) the system of equations (5) can be rewritten in the following form:

$$
\begin{aligned}
{\left[1-\alpha \hat{f}\left(\mathbf{k}_{0}\right)\right] \mathbf{E}_{l}^{0}=} & \mathbf{E}_{I l}-i 2 \pi C a \sum_{j=1}^{N}, \sum_{p, q=-\infty}^{\infty}\left[\mathbf{k}_{p q} \times\left(\mathbf{k}_{p q} \times \mathbf{E}_{j}^{0}\right)\right] \frac{\exp \left[i\left(\mathbf{k}_{p q}-\mathbf{k}_{0}^{\|}\right)\left(\mathbf{r}_{l}^{0}-\mathbf{r}_{j}^{0}\right)\right]}{\kappa_{p q}} \\
& l=\overline{1, N},
\end{aligned}
$$

where $C=\alpha /\left(a\left|\mathbf{a}_{1} \times \mathbf{a}_{2}\right|\right)$,

$$
\mathbf{E}_{I l}=\mathbf{E}_{0 I} \exp \left(i \mathbf{k}_{0}^{\perp} \mathbf{r}_{a_{l}}\right)=\mathbf{E}_{0 I} \exp \left(-i k_{0} \cos \Theta_{I} z_{l}\right), \quad l=\overline{1, N} .
$$

Prime at the sum sign in (33) means that the summand with $j=l$ is excluded.

The results of numerical solution of the system of equations (33) for the cubic lattice and $s$-polarized incident wave are shown in Fig. 1. In order to demonstrate to the fullest extent the characteristic features of the field behavior near the film surface we have used a rather high value of the volumic polarizability $(C=0.2)$. Normally in the optical range $C=0.04 \div 0.15$ which corresponds to the refractive index $n=1.27 \div 2.46$ [39]. However, for some materials $C$ can be higher. For instance, $n_{G a P}\left(\lambda_{0}=564 \mathrm{~nm}\right)=3.42$ [23, 40] and $n_{S i}\left(\lambda_{0}=620 \mathrm{~nm}\right)=3.94$ [22, 40] which corresponds to $C_{G a P}=0.19$ and $C_{S i}=0.2$, respectively.

In Fig. 1 the results of the calculations according to the Airy formulae are also shown for comparison. Besides we have used the Airy formulae for two cases: (1) the film occupies the region of space $-a(N-1)<z<0$, and (2) the film occupies the region of space $-a(N-1 / 2)<$ $z<a / 2$. In the former case the corresponding formula has the form 33.

$$
\begin{gathered}
E^{y}(\mathbf{r})=\frac{t_{\perp}\left[\exp \left(i k_{0} n \mathbf{s}_{T}^{(-)} \mathbf{r}\right)-r_{\perp} \exp (i \varphi) \exp \left(i k_{0} n \mathbf{s}_{T}^{(+)} \mathbf{r}\right)\right]}{1-r_{\perp}^{2} \exp (i \varphi)} \frac{E_{0 I}^{y}}{1-(4 \pi / 3) C} \\
\mathbf{s}_{T}^{( \pm)}=\left(-\sin \Theta_{T}, 0, \pm \cos \Theta_{T}\right), \varphi=2 k_{0} h n \cos \Theta_{T}
\end{gathered}
$$



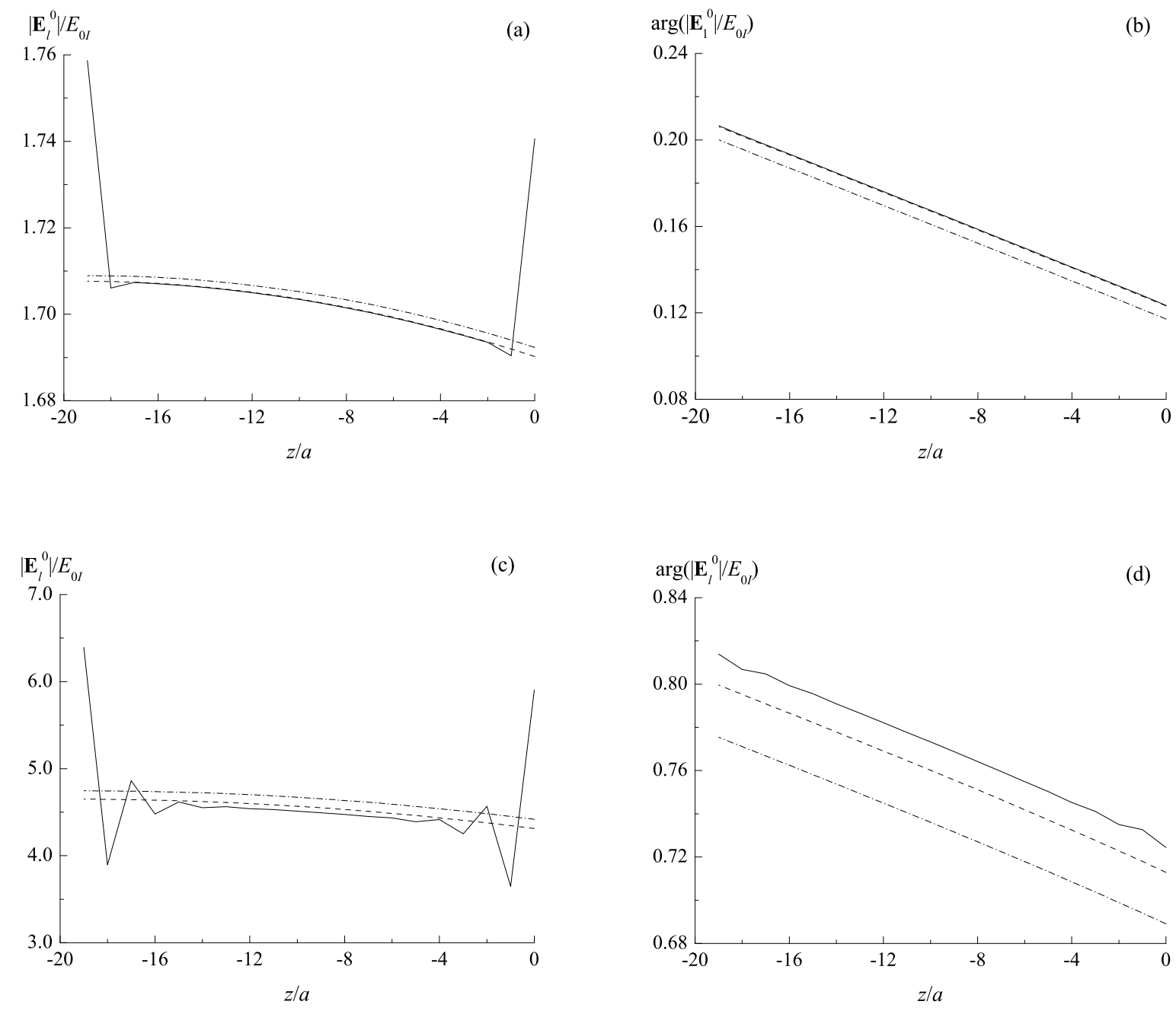

Figure 1: Coordinate dependences of the amplitude (a, c) and the phase (b, d) of the local electric field strength inside the film: — numerical solution of the system of equations (33), - - - calculation according to the Airy formulae for the case when the film occupies the region of space $-a(N-1)<z<0,--$ calculation according to the Airy formulae for the case when the film occupies the region of space $-a(N-1 / 2)<z<a / 2$. The calculations are performed for the cubic lattice. The external wave is polarized along one of the translation vector of the lattice (s-polarization). The parameters are: $k_{0}=0.01 \mathrm{~nm}^{-1}, a=0.5 \mathrm{~nm}, N=20$, $\Theta_{I}=30^{\circ}, C=0.1(\mathrm{a}, \mathrm{b}) ; C=0.2(\mathrm{c}, \mathrm{d})$. 
where $\Theta_{T}$ is the angle of refraction, $t_{\perp}$ and $r_{\perp}$ are Fresnel coefficients of transmittance and reflectance for s-polarization, respectively, $h=a(N-1)$ is a film thickness, $n$ is a refractive index defined by the Lorentz-Lorenz relation (18).

In the latter case $h=a N$, and in eq.(35) one has to make the replacement $\mathbf{r} \rightarrow \mathbf{r}-$ $\mathbf{n} a / 2, E_{0 I}^{y} \rightarrow E_{0 I}^{y} \exp \left(-i k_{0} a \cos \Theta_{I} / 2\right)$. It seems that the first form of the Airy formulae better corresponds to the initial statement of the problem. However, the results of numerical solution of the system (33) are in a better agreement with the second form. The reason is that in the former case the macroscopic density $\rho$ which enters the Airy formulae appears to be higher than its true value $\left(a\left|\mathbf{a}_{1} \times \mathbf{a}_{2}\right|\right)^{-1}$, but in the latter case $\rho$ in the Airy formulae is equal to $\left(a\left|\mathbf{a}_{1} \times \mathbf{a}_{2}\right|\right)^{-1}$ as it should be.

As it follows from the numerical calculations, the consideration of the discrete structure influences the most significantly the behavior of the field amplitude near the film surface where rapid oscillations of the local field take place. The amplitude of these oscillations and the penetration depth into the film volume increase with the parameter $C$. One can also see on Fig. 1 that the consideration of the discrete structure does not influence much the phase of the field. This means that the wave vector of the field remains almost the same at any point inside the film. Therefore, the real part of the refractive index of the field is constant. Far from the boundaries the distribution of the field is regular and approximately coincides the results given by the Airy formulae.

\section{$5 \quad$ Field on the probe}

When the system of equations (33) is solved and all the quantities $\mathbf{E}_{j}^{0}, j=\overline{1, N}$ are determined, one can calculate the field at the probe position:

$$
\mathbf{E}(\mathbf{r})=\mathbf{A}_{I}(\mathbf{r})-2 \pi i C a \sum_{j=1}^{N} \sum_{p, q=-\infty}^{\infty}\left[\mathbf{k}_{p q} \times\left(\mathbf{k}_{p q} \times \mathbf{E}_{j}^{0}\right)\right] \frac{\exp \left[i \mathbf{k}_{p q}\left(\mathbf{r}-\mathbf{r}_{j}^{0}\right)\right]}{\kappa_{p q}} \exp \left(i \mathbf{k}_{0}^{\|} \mathbf{r}_{j}^{0}\right) .
$$

Under the condition (15) the field $\mathbf{E}(\mathbf{r})$ equals to the field $\mathbf{E}_{p}$ acting on the probe. Let's analyze in details the expression (37).

\subsection{Field in the wave zone}

Let's consider first the case when the observation point is in the wave zone, i.e., $\left|z-z_{j}\right| \rightarrow \infty$. In this case the summands with $p, q \neq 0$ do not contribute to the resultant field due to the following reason. All the quantities $g_{p q}^{\|}$at $p, q \neq 0$ are of the order of $2 \pi / a$ and they are much greater than $k_{0}$ if $k_{0} a \ll 1$. Therefore, $\kappa_{p q}$ (27) are purely imaginary and all the corresponding summands in (25) decay rapidly with the increase of the distance from the surface. Only the term with $\mathbf{k}_{00}$ survives. If $z>z_{j}, \mathbf{k}_{00}$ represents itself the wave vector of the reflected wave $\mathbf{k}_{R}$, otherwise $\mathbf{k}_{00}=\mathbf{k}_{0}$. Therefore, in the wave zone we have plane propagating waves:

$$
\begin{aligned}
& \mathbf{E}(\mathbf{r})=\left\{\begin{aligned}
\mathbf{A}_{I}(\mathbf{r})+\mathbf{E}_{R}^{\text {far }} \exp \left(i \mathbf{k}_{R} \mathbf{r}\right), & z>0 ; \\
\mathbf{E}_{T}^{f a r} \exp \left(i \mathbf{k}_{0} \mathbf{r}\right), & z<-a(N-1) ;
\end{aligned}\right. \\
& \mathbf{E}_{R}^{f a r}=-i 2 \pi C \frac{a}{k_{0} \cos \Theta_{I}} \sum_{j=1}^{N}\left[\mathbf{k}_{R} \times\left(\mathbf{k}_{R} \times \mathbf{E}_{j}^{0}\right)\right] \exp \left(-i \mathbf{k}_{R}^{\perp} \mathbf{r}_{j}^{0}\right), \\
& \mathbf{E}_{T}^{\text {far }}=\mathbf{E}_{0 I}-i 2 \pi C \frac{a}{k_{0} \cos \Theta_{I}} \sum_{j=1}^{N}\left[\mathbf{k}_{0} \times\left(\mathbf{k}_{0} \times \mathbf{E}_{j}^{0}\right)\right] \exp \left(-i \mathbf{k}_{0}^{\perp} \mathbf{r}_{j}^{0}\right) .
\end{aligned}
$$




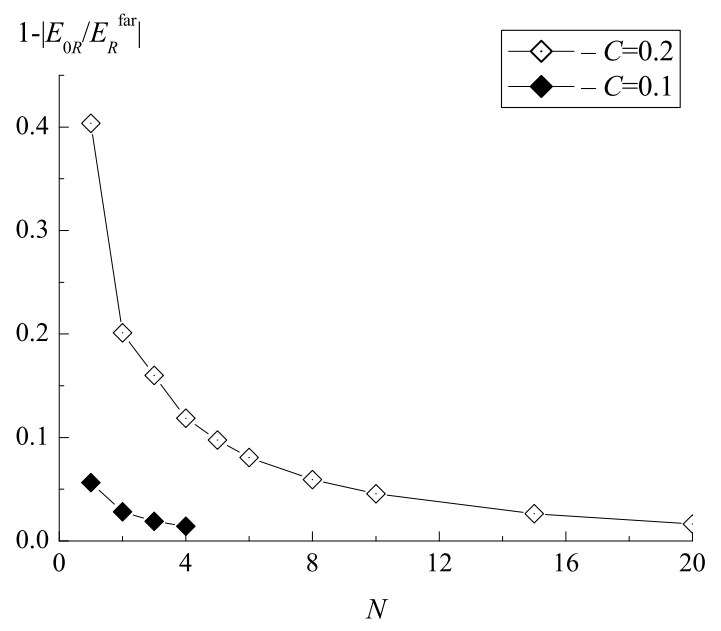

Figure 2: The difference between the reflected wave field in the near zone, calculated under $\left(E_{R}^{f a r}\right)$ and without $\left(E_{0 R}\right)$ the consideration of the discrete structure of the medium, as a function of the number of monolayers $N$ which constitute the film. The calculations are performed for the cubic lattice. The parameters are: $k_{0}=0.01 \mathrm{~nm}^{-1}, a=0.5 \mathrm{~nm}, \Theta_{I}=0^{o}$.

This fields are transverse, because $\mathbf{k}_{R} \mathbf{E}_{R}^{f a r}=\mathbf{k}_{0} \mathbf{E}_{T}^{f a r}=0$. The amplitudes $\mathbf{E}_{R}^{f a r}, \mathbf{E}_{T}^{f a r}$ do not depend on the coordinates.

The discrepancy between the values of the field of the reflected wave calculated under consideration of the discrete structure and according to the Airy formulae can be of the order of $10 \%$. This discrepancy becomes larger with the decrease of the film thickness and with the increase of the parameter $C$ (Fig. 2). The plots on Fig. 2 lead to the conclusion that at high enough film thicknesses $(N \geq 20)$ the Airy formulae give a satisfactory description of the reflected waves in the wave zone. In this situation our method of calculation becomes equivalent to that developed in Refs. [25, 26, 27]. As for the dependence on the incident angle, the maximal discrepancy between the amplitudes of the reflected wave calculated under consideration and without consideration of the discrete structure is observed at normal incidence. With the increase of the incident angle this discrepancy gradually vanishes (Fig. 3). In the calculations presented on Figs. 2, 3 we used the Airy formulae for the case when the film occupies the region of the space $-a(N-1 / 2)<z<a / 2$ [33]

$$
E_{0 R}^{y}=\frac{r_{\perp}[1-\exp (i \varphi)]}{1-r_{\perp}^{2} \exp (i \varphi)} \exp \left(-i k_{0} a \cos \Theta_{I}\right) E_{0 I}^{y} .
$$

Here we use the same notations as in formula (35). Besides, as for the field inside the film, the results of our calculations are in a better agreement with the Airy formulae compared to the case when the film occupies the region of space $-a(N-1)<z<0$. We would like to note also that for $p$-polarized wave the field of the reflected wave near the Brewster angle does not vanish 41].

\subsection{Field in the near zone}

If the observation point is near the surface, one can observe a number of characteristic features in the field behavior which we are going to discuss now. For the sake of simplicity we shall restrict 


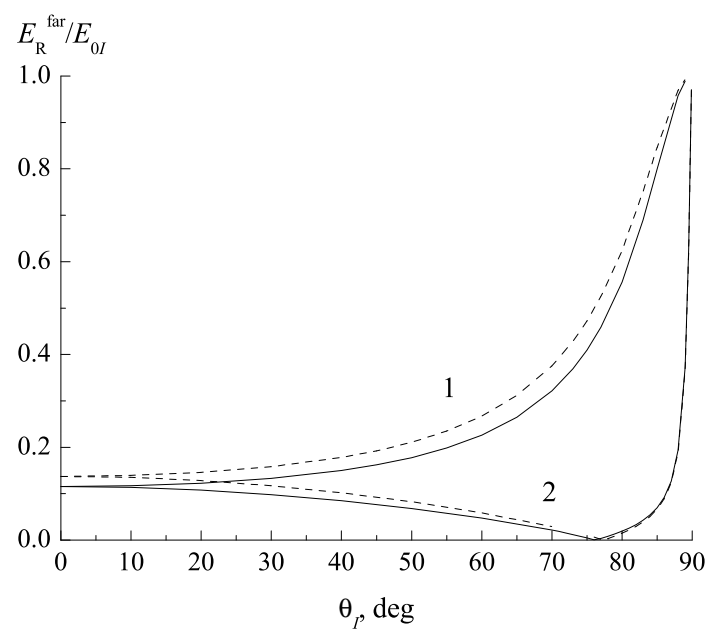

Figure 3: The dependence of the electric field strength amplitude of the reflected wave $E_{R}^{f a r}$ on the incident angle of the external wave $\Theta_{I}$ in the wave zone for $s_{-}(1)$ and p- $(2)$ polarizations: — under consideration of the discrete structure, - - - according to the Airy formulae. The calculations are performed for the cubic lattice. The parameters are: $k_{0}=0.01 \mathrm{~nm}^{-1}$, $a=0.5 \mathrm{~nm}, N=3, C=0.2$.

ourselves, if the opposite is not stated explicitly, by the case of quadratic lattice $\left(\mathbf{a}_{1} \perp \mathbf{a}_{2}\right.$, $\left.a_{1}=a_{2}=a ; g_{1}=g_{2}=g=2 \pi / a\right)$.

\subsubsection{Contributions of propagating and evanescent harmonics}

At a small distance from the surface the summands with $p, q \neq 0$ in (37) can make a noticeable contribution. Let's estimate this contribution considering the following example. As it was mentioned above in the discussion of formula (25), it is convenient to single out in eq.(37) evanescent waves $\mathbf{E}_{\kappa}=\sum_{p^{2}+q^{2}=\kappa} \mathbf{A}_{p q} \exp \left(i \mathbf{k}_{0}^{\|} \mathbf{r}\right)$ with the decay coefficients dependent on $\kappa$. The ratio of the amplitudes of the evanescent harmonics $E_{\kappa}$ for $\kappa=0$ (plane reflected wave), 1, 2, 4 is shown on Fig. 4 . Note that the relative contribution of different $E_{\kappa}$ strongly depends on the distance between the probe and the film surface. The behavior shown on Fig. 4 is typical for the distances greater or equal to one lattice constant. Usually if one takes into account the modes with $p, q=0, \pm 1, \pm 2$ the tolerance of the calculations is less than one per cent. As it follows from the dependences on Fig. 4, at $k_{0} a=\pi$ (a typical value for photonic crystals and optical lattices) the monolayers do not fill the evanescent waves radiated by the other monolayers. At $z=2 a$ and $k_{0} a=0.005$ the ratio of decaying and non-decaying field components is about $1 \%$ and rapidly decreases with the increase of $k_{0} a$. If $a \ll 1 / k_{0}$, then $g \gg k_{0}$, and as it follows from eqs.(25)-(27) the form of the evanescent waves is almost independent of the incidence angle of the external wave. Coordinate dependences of the total amplitude of the evanescent harmonics is shown on Fig. 5.

The dependence of the distance $L_{e}$, at which the amplitudes of the evanescent and propagating components are equal, on the parameter $k_{0} a$ is depicted on Fig. 6. Due to the rapid decay of the evanescent harmonics the quantity $L_{e}$ can be treated as a distance from the surface up to which the contribution of the evanescent component to the resultant field is essential. From 


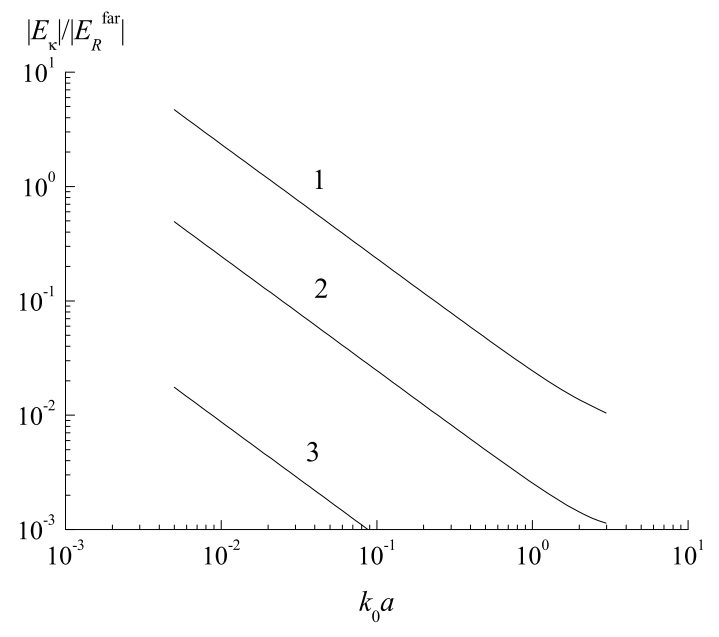

Figure 4: The ratio of the evanescent field amplitudes near the surface $E_{\kappa}$ to the amplitude of the reflected field in the wave zone $E_{R}^{f a r}$. The calculations are performed for the cubic lattice. The parameters are: $k_{0}=0.01 \mathrm{~nm}^{-1}, \Theta_{I}=0$, the distance between the observation point and the surface is equal to $a .1-\left|E_{1} / E_{R}^{f a r}\right|, 2-\left|E_{2} / E_{R}^{\text {far }}\right|, 3-\left|E_{4} / E_{R}^{\text {far }}\right|$.

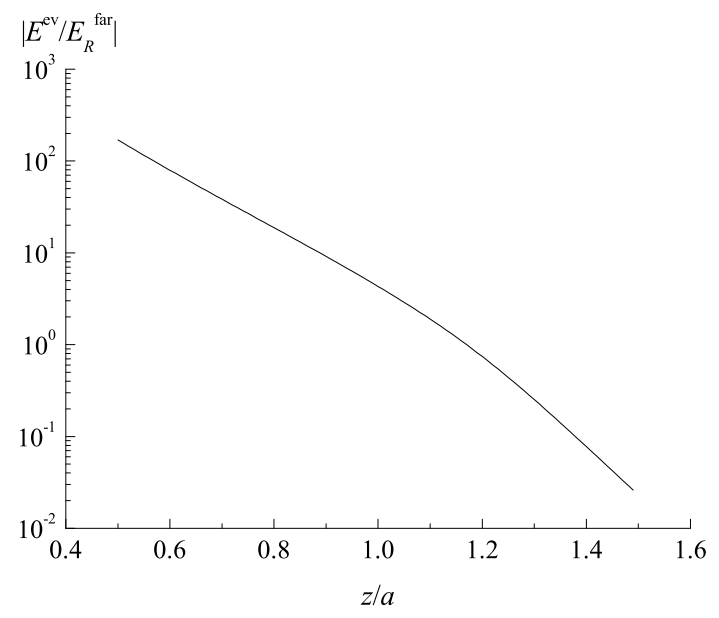

Figure 5: Coordinate dependences of the ratio of the total amplitude of the evanescent waves $E^{e v}$ to the amplitude of the reflected wave field in the wave zone $E_{R}^{f a r}$. The calculations are performed for the cubic lattice. The parameters are: $k_{0}=0.01 \mathrm{~nm}^{-1}, a=0.5 \mathrm{~nm}, \Theta_{I}=0^{o}$. The observation point is above an atom of the film. 


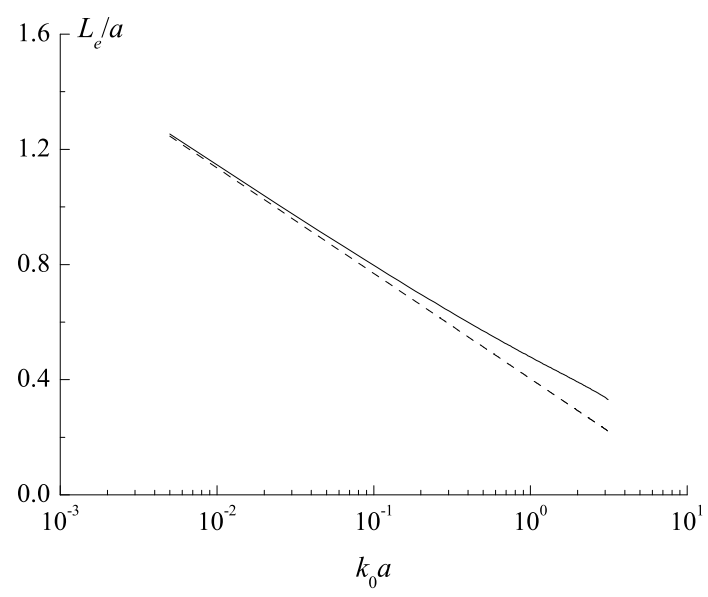

Figure 6: The dependence of the distance $L_{e}$, at which the amplitudes of the evanescent and propagating parts of the reflected wave field are equal to one another, on the parameter $k_{0} a$ : - exact numerical calculations, $-\quad-\quad$ an estimate according to formula (42). The calculations are performed for the cubic lattice, $\Theta_{I}=0^{\circ}$.

the dependence shown on Fig. 6 it follows that the contribution of the evanescent component is essential at the distances less than two lattice constants. The field radiated by the monolayers, which are located at larger distances from the observation point, can be treated as propagating plane waves.

The distance $L_{e}$ can be also analytically estimated on the basis of the following consideration. As it follows from the dependences depicted on Fig. the leading contribution to the decaying wave is made by the evanescent wave with $\kappa=1$. Therefore, the distance $L_{e}$ can be approximately determined from the condition

$$
\frac{\left|E_{1}\right|}{\left|E_{R}^{f a r}\right|}=1
$$

In the case $k_{0} a \ll 1$ we have $\mathbf{k}_{p q} \approx\left(\mathbf{g}_{p q}^{\|}, i g_{p q}^{\|}\right)$. Then for a monolayer we can write the condition (40) anew as

$$
2 \frac{2 \pi}{k_{0} a} \exp \left(-2 \pi \frac{L_{e}}{a}\right)=1
$$

from which we get

$$
\frac{L_{e}}{a}=\frac{1}{2 \pi} \ln \left(\frac{4 \pi}{k_{0} a}\right) .
$$

As it follows from the dependences presented on Fig. 6, the estimate (42) is in a good agreement with the results of exact numerical calculations at small $a$.

We can approximately estimate the distance $L_{e}$ for a semi-infinite medium in a similar manner assuming that the field of the reflected wave in the wave zone and the field of the transmitted wave in the medium are defined by Fresnel reflection and transmission coefficients $r_{F}$ and $t_{F}$, respectively. Then for $s$-polarized external wave after minor algebra we get

$$
\frac{L_{e}}{a}=\frac{1}{2 \pi} \ln \left|\frac{8 \pi^{2} C t_{F}^{s}}{r_{F}^{s}\left(1-\frac{4 \pi}{3} C\right)}\right| .
$$


In the case of the normal incidence $r_{F}^{s}=-(n-1) /(n+1)$ and $t_{F}^{s}=2 /(n+1)$. Taking into account the Lorentz-Lorenz formula (18), we obtain

$$
\frac{L_{e}}{a}=\frac{1}{2 \pi} \ln [4 \pi(n+1)]
$$

From eq.(44) it folows that if the refractive index $n$ is in the range $1.1 \div 4.0$, then $L_{e} / a$ is in the range $0.5 \div 0.7$. In fact $L_{e} / a$ has to be higher, because as it follows from the numerical calculations in section 4 the local field inside the film in the first atomic layer is higher than that in the "bulk".

In the case of $p$-polarized external wave incident on a semi-infinite medium the estimation of $L_{e}$ will be given by an equation which has similar structure as (43) with $t_{F}^{s}$ and $r_{F}^{s}$ replaced by $t_{F}^{p}$ and $r_{F}^{p}$, respectively. In this case near the Brewster angle the reflection can be rather small but still noticeable. In such a regime we get the values of $L_{e} / a$ which are somewhat bigger than 1. Therefore, our estimations show that the propagating and evanescent harmonics can make comparable contributions to the optical response of the medium at the distances from the surface of the order of one lattice constant.

\subsubsection{Non-transversality of the total fields of reflected and transmitted waves}

In distinction to the wave zone the polarization vector of the field in the near zone has no a definite direction. Its magnitude and the direction depend on all three spatial coordinates. Indeed, the polarization vector of a certain evanescent harmonic in the r.h.s of eq.(37) is perpendicular to the wave vector $\mathbf{k}_{p q}$, which in its turn has different orientations depending on $p$ and $q$. In addition, each harmonic has its own decay coefficient $\kappa_{p q}$ and the behavior of the harmonic along the surface is defined by its own vector $\mathbf{g}_{p q}^{\|}$. Carrying out the summation over all the harmonics we get the properties of the polarization vector mentioned above. In particular, in the case of the normal incidence of the external wave with the polarization vector along the $y$ axis the field near the film surface has non-vanishing $x$ - and $z$-components (Fig. 7a,c).

For the purposes of the further analysis let's estimate the ratio of the evanescent component of the reflected wave field to the incident wave field. From the Airy formulae for the $s$-polarization it follows that in the case of ultrathin film the local field inside the film is $E_{0} \approx E_{0 I} /[1-(4 \pi / 3) C]$. The evanescent field at the distance of one lattice constant from the surface is mainly determined by the harmonic $E_{1}$. For the square lattice $E_{1}=$ $-2(2 \pi)^{2} C \exp (-2 \pi) E_{0} \approx 0.147 C /[1-(4 \pi / 3) C] E_{0 I}$. Even for $C=0.2$ the ratio $E_{1} / E_{0 I} \approx 0.2$. Thus, the amplitude of the evanescent wave at the distance from the surface of the order of one lattice constant is usually much less than the incident field amplitude. Therefore, one can conclude that in the field of the probe the main contribution is made by the component of the field directed along the polarization vector of the external wave. In particular, comparing the intensities of $x$ and $z$-components of the field (Fig. 7a,c) with $y$-component (Fig. 7b) one can notice that the contribution of $x$ - and $z$-components is small and the field behavior near the surface is mainly determined by $y$-component.

One can come to one more conclusion regarding the direction of the field polarization vector near the film surface. Under the normal incidence of the external wave in the case when there are no parallel shifts of the atomic planes relative to one another, i.e., $\alpha_{1 j}=\alpha_{2 j}=0, j=\overline{1, N}$, at the observation points above the atoms the $z$-component of the reflected wave field vanishes (Fig. 7). Indeed, taking into account the properties of the tensor $\hat{f}$ and summing in (33) the harmonics with equal $\kappa_{p q}\left(\kappa_{p q}=\kappa_{-p,-q}\right)$, we get that $\left(\mathbf{E}_{j}^{0}\right)_{x, y}$ are independent of $\left(\mathbf{E}_{j}^{0}\right)_{z}$. The $z$ 

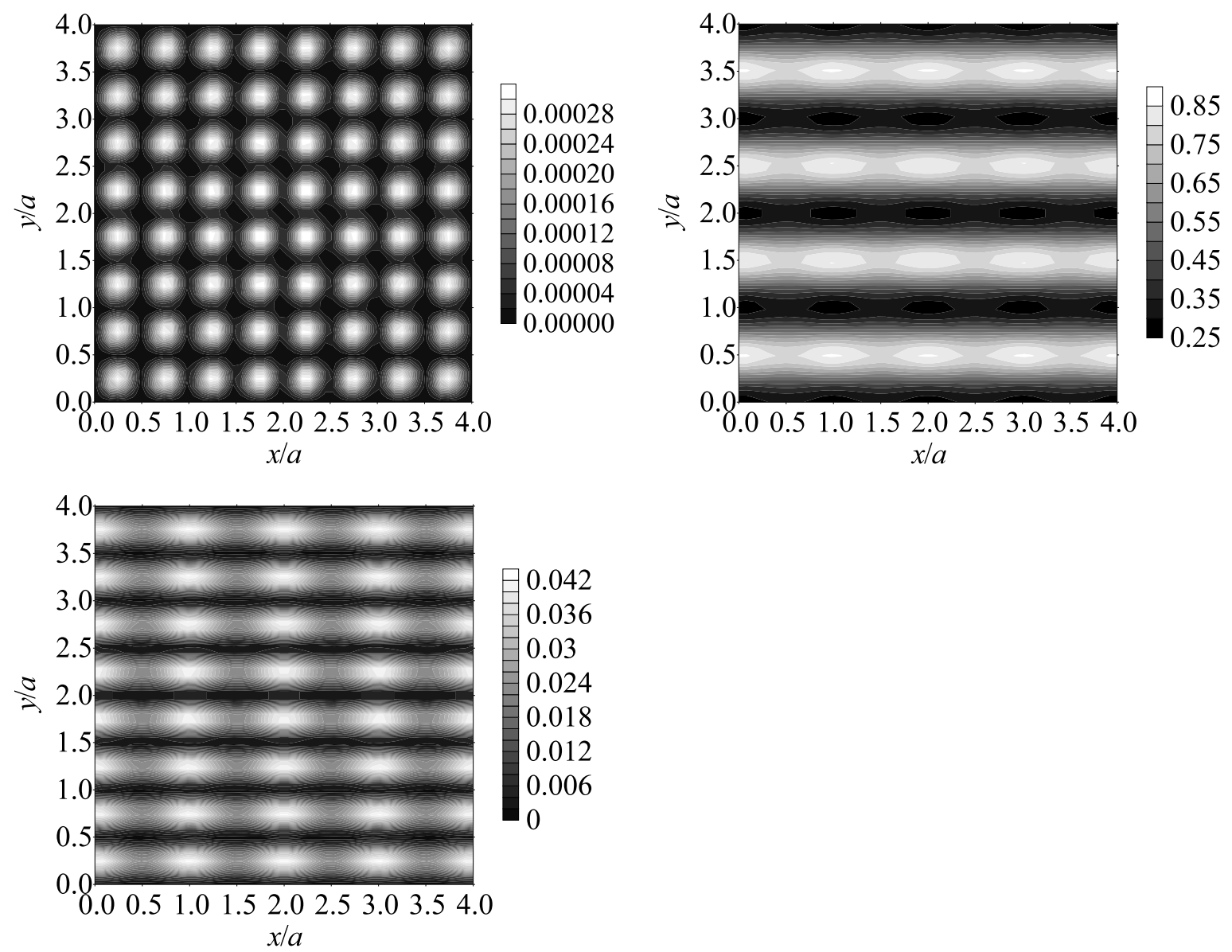

Figure 7: The ratio of the intensities of $x$ - (a), $y$ - (b) and $z$ - (c) components of the electromagnetic field on the probe $\left(I_{x} \sim\left|E_{x}\right|^{2}, I_{y} \sim\left|E_{y}\right|^{2}, I_{z} \sim\left|E_{z}\right|^{2}\right)$ to the intensity of the incident wave $I_{0}$ on the coordinates $x$ and $y$. The parameters are: the number of monolayers in the film $N=20, C=0.2, k_{0}=0.01 \mathrm{~nm}^{-1}, a=0.5 \mathrm{~nm}, d=a, \Theta_{I}=0^{\circ}, \Phi_{I}=0^{\circ}$. The intensity minima correspond to the atomic positions. In spite of the fact that the external wave is polarized along the y axis, optical responds of the medium contains all three components. 
components of the field inside the film satisfy to the system of homogeneous equations which has only the trivial solution $\left(\mathbf{E}_{j}^{0}\right)_{z} \equiv 0, j=\overline{1, N}$. Then it follows immediately from (37) that at the observation points above the atoms $\left(\mathbf{E}_{p}\right)_{z}=0$.

\subsubsection{Dependence of field intensity on the longitudinal coordinates $x$ and $y$}

The electromagnetic field intensity is defined by its strength squared. In a general case it can be calculated numerically. But in the case of the normal incidence $\left(\Theta_{I}=0\right)$ one can obtain rather simple analytical expressions. Let's consider this case. From the plots on Fig. 6 it follows that the component of the field, which is a periodic function of longitudinal coordinates, is induced mainly by the surface atoms. The bulk atoms produce only a constant background. Thus, in order to analyze the behavior of the evanescent waves it is enough to consider only one surface monolayer $\left(z_{j}=0\right.$ in eq.(37)). For the qualitative analysis of the field behavior near the film surface it is enough to keep in eq.(37) only the harmonics with $(p, q)=(1,0)$, $(-1,0),(0,1),(0,-1)$ (we assume that the quantities $g_{1}$ and $g_{2}$ differ from one another no more that two times). The amplitudes of all other harmonics are at least one order of magnitude less [42]. Omitting the unimportant constant factor after an elementary algebra we obtain an approximate expression for the field intensity at the probe position:

$$
\begin{aligned}
I & \approx\left[E_{0 I}+\operatorname{Re}\left(E_{R}^{f a r}\right)\right. \\
& \left.-4 \pi C a \sum_{i=1,2} \frac{\left(\mathbf{g}_{i} \mathbf{E}_{0 I}\right)}{E_{0 I}}\left\{\operatorname{Re}\left(\mathbf{g}_{i} \mathbf{E}_{1}^{0}\right) \cos \left[\mathbf{g}_{i}\left(\mathbf{r}-\mathbf{r}_{1}^{0}\right)\right]-g_{i} \operatorname{Re}\left(E_{1 z}^{0}\right) \sin \left[\mathbf{g}_{i}\left(\mathbf{r}-\mathbf{r}_{1}^{0}\right)\right]\right\} \frac{\exp \left(-g_{i} z\right)}{g_{i}}\right]^{2},
\end{aligned}
$$

which is valid for the films of arbitrary thickness. Here $E_{1}^{0}$ is an amplitude of the unperturbed part of the field in the first (surface) monolayer. In fact, this expression is the second power of the real part of the field component at the probe position, directed along the polarization vector of the external field. The imaginary part of this component and all other components are negligible.

From the expression (45) one can see that in general the intensity minima and maxima are shifted relative to the atomic positions. This is due to the term which contains $E_{1 z}^{0}$. Due to the fact that even in the case of the normal incidence the dependence of the direction of the vector $\mathbf{E}_{1}^{0}$ on the direction of $\mathbf{E}_{0 I}$ is rather complicated, the location of the intensity minima and maxima depends on $\mathbf{E}_{0 I}$ in a complicated manner as well. Let's consider the case when there are no parallel shifts of the atomic planes relative to one another. As it was shown above, in this case $E_{1 z}^{0}=0$ and the expression (45) becomes simpler. In this case the intensity minima are located exactly at the positions of the atoms. Indeed, at these points $\cos \left[\mathbf{g}_{1}\left(\mathbf{r}-\mathbf{r}_{1}^{0}\right)\right]=\cos \left[\mathbf{g}_{2}\left(\mathbf{r}-\mathbf{r}_{1}^{0}\right)\right]=1$. Thus, formula (45) explains the contrast reversal, which has been obtained in Refs. [36, 43] by means of numerical calculations.

In analogous manner one could assume that the intensity maxima are located in the centers of the elementary cells, where the cosines are equal to -1 . However, this is not exactly the case as one can see on Fig. 8. With the change of the mutual orientation of the polarization vector of the external wave and the lattice elementary translations vectors the location of the intensity maxima changes as well. The reason is the following. Let the polarization vector of the external wave is perpendicular to one of the vectors of the reciprocal lattice, for instance, $\left(\mathbf{E}_{0 I} \mathbf{g}_{1}\right)=0$. The field behavior along the direction $\mathbf{a}_{1}$ in this case is determined by higher order harmonics with the smallest decay coefficient and with non-vanishing component along 

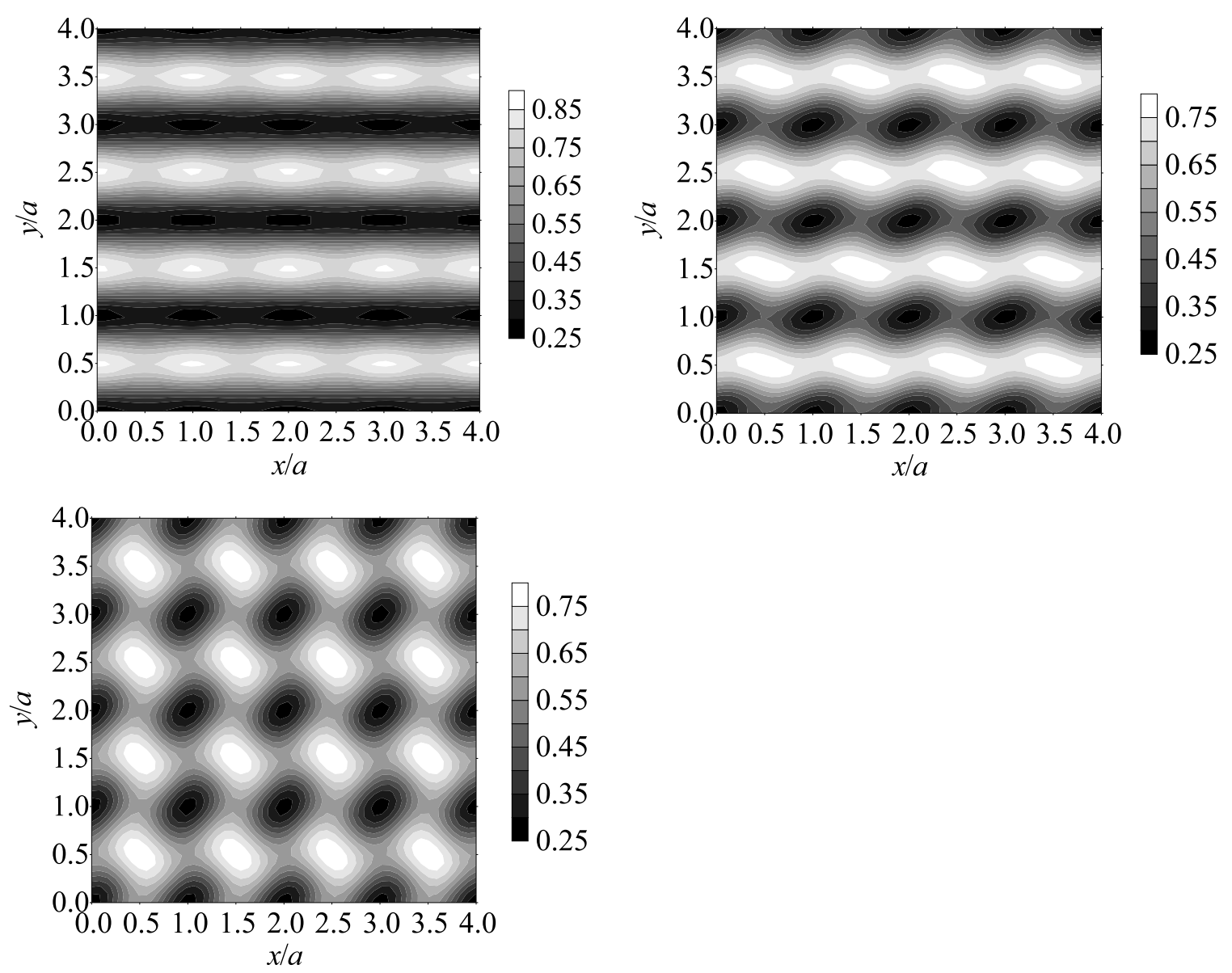

Figure 8: The ratio of the electromagnetic field intensity on the probe $I$ to the incident wave intensity $I_{0}$. The parameters are: $N=20, C=0.2, k_{0}=0.01 \mathrm{~nm}^{-1}, a=0.5 \mathrm{~nm}, d=a$, $\Theta_{I}=0^{\circ} ; \Phi_{I}=0^{\circ}(\mathrm{a}), 30^{\circ}(\mathrm{b}), 45^{\circ}(\mathrm{c})$. The electromagnetic field intensity is a periodic function of the coordinates $x$ and $y$, and the period is equal to the lattice constant $a$. The intensity minima correspond to the atomic positions. 
the polarization vector of the external wave. Obviously, these are harmonics with $|p|=|q|=1$. In eq.(45) in the square brackets one has to add one more term

$$
-4 \pi C a \frac{\left(\mathbf{g}_{11}^{\min } \mathbf{E}_{0 I}\right)}{E_{0 I}} \operatorname{Re}\left(\mathbf{g}_{11}^{\min } \mathbf{E}_{1}^{0}\right) \cos \left[\mathbf{g}_{11}^{\min }\left(\mathbf{r}-\mathbf{r}_{1}^{0}\right)\right] \frac{\exp \left(-g_{11}^{\min } z\right)}{g_{11}^{\min }}
$$

where $\mathbf{g}_{11}^{\min }$ is one of the vectors $\mathbf{g}_{p q}$ with $|p|=|q|=1$, which has the minimal length. At the observation points above the atoms $\left(\mathbf{r}^{\|}=\mathbf{r}_{a_{1}}^{\|}\right)$this term is minimal, i.e., the intensity minima are still above the atoms, even provided that $\left(\mathbf{E}_{0 I} \mathbf{g}_{1}\right)=0$ or $\left(\mathbf{E}_{1}^{0} \mathbf{g}_{1}\right)=0$. The locations of the intensity maxima can be found from the condition $\left(\mathbf{g}_{11}^{\min } \mathbf{r}\right)=\pi \pm 2 \pi k$, where $k$ is an integer. This condition fulfills not in the centers of the elementary cells, like in the case when the directions of the polarization vector of the external wave $\mathbf{E}_{0 I}$ and the local field inside the film $\mathbf{E}_{1}^{0}$ does not coincide with any of the translation vectors of the lattice (Fig. 8 b,c), but at the points $\mathbf{r}^{\|}=\mathbf{r}_{a_{1}}^{\|}+\mathbf{a}_{1} / 2$ or $\mathbf{r}^{\|}=\mathbf{r}_{a_{1}}^{\|}+\mathbf{a}_{2} / 2$ (Fig. 8a). Thus, in distinction to the intensity minima locations, which are always above the atoms, the locations of the intensity maxima depend on the mutual orientation of the field polarization and the translation vectors of the lattice.

Formula (45) allows one to investigate the image contrast, which is defined here as a maximal difference of the light intensity along a certain direction. In Refs. [4, 36, 44] it was point out that the field component parallel to the scan direction displays a better contrast than that perpendicular to this direction. Let's consider this problem in more details. The image contrast in the direction $\mathbf{a}_{i}$ is determined by the mutual orientation of the vectors $\mathbf{E}_{0 I}, \mathbf{E}_{1}^{0}$, and $\mathbf{g}_{i}$. The maximal contrast is reached at some angle between $\mathbf{E}_{0 I}$ and $\mathbf{g}_{i}$ when the coefficient $\left(\mathbf{g}_{i} \mathbf{E}_{0 I}\right) \operatorname{Re}\left(\mathbf{g}_{i} \mathbf{E}_{1}^{0}\right)$ takes the largest value. This angle depends in general on the symmetry of the atomic distribution at the surface and on the atomic polarizability. We are going to come back to this issue in our subsequent publications.

The minimal contrast along the direction $\mathbf{a}_{1}$ for the lines which pass through the atoms is achieved when the polarization vector of the external wave or the field vector in the film is perpendicular to the vector of the reciprocal lattice $\mathbf{g}_{1}$ (parallel to the vector of the real lattice $\mathbf{a}_{2}$ ) (45). Performing numerical calculations, one can show that in general the conditions $\left(\mathbf{g}_{i} \mathbf{E}_{0 I}\right)=0$ and $\left(\mathbf{g}_{i} \mathbf{E}_{1}^{0}\right)=0$ are satisfied at different directions of the polarization vector of the external wave. Thus, in general there exist two angles at which contrast minima can be observed. The angle between the two directions of the polarization vector of the external field allows one to get the information about the local field inside the film and, therefore, about the atomic polarizability.

Another situation occurs for the contrast along the lines which pass through the centers of the elementary cells. In this case the contrast minima are determined by the condition of mutual compensation of the contributions from the harmonics with decay coefficients $g_{1}$ and $g_{11}^{\min }$. The compensation occurs at some angle $\phi_{c}$ between the polarization vector of the field inside the film and the vector $\mathbf{g}_{1}$, which depends on the symmetry of the atomic distribution at the surface. The angle $\phi_{c}$ strongly depends on the distance from the surface.

Let's consider the simplest case of the cubic lattice. Under the normal incidence of the external wave the direction of the polarization vector of the field inside the film coincides with that of the external field. In this case there are two directions of the polarization vector of the external wave, for which one can observe the contrast minima along the lines which pass through the atoms. This corresponds to the situations when the polarization vector coincides with $\mathbf{a}_{1}$ or $\mathbf{a}_{2}$. For the cubic lattice one can get an explicit expression for the angle $\phi_{c}$ at which one observes the minimal contrast along the lines which pass through the centers of the cells. The dependence of the angle $\phi_{c}$ on the distance between the observation point and the surface 


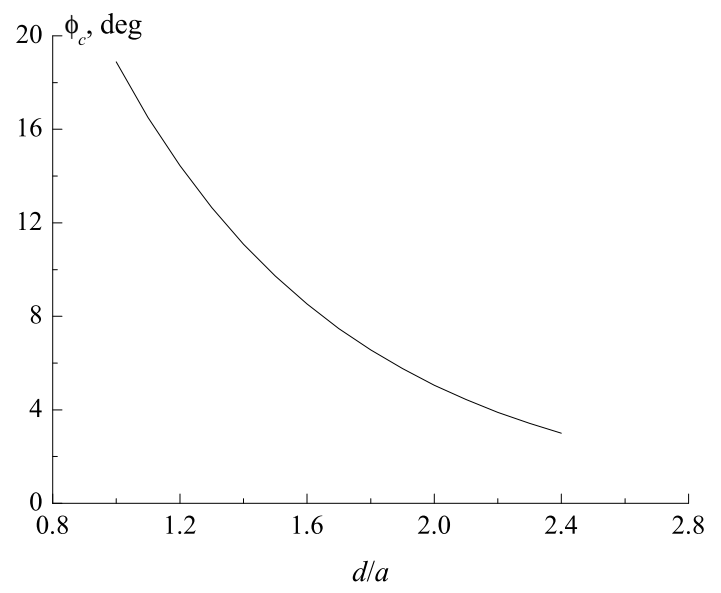

Figure 9: The angle between the minimal contrast direction and the polarization vector of the external wave $\phi_{c}$ as a function of the distance to the film surface $d$. The calculations are performed for the cubic lattice, $\Theta_{I}=0^{\circ}$.

is given by the approximate formula

$$
\sin \phi_{c}=2^{1 / 4} \exp [-\pi(\sqrt{2}-1) d / a]
$$

which is valid only for $d \geq a$. The dependence $\phi_{c}(d)$ for the cubic lattice is shown on Fig. 9. As one can see on the figure, the angle $\phi_{c}$ can take the values of dozens of degrees at the distance from the surface of the order of one lattice constant. The magnitude of the angle remains essential at the distances of several lattice constants.

Thus, changing the direction of the polarization vector of the external wave one can manipulate the image contrast. One can determine the atomic positions and the symmetry of the crystal lattice detecting the intensity minima. Measuring the angle between the directions of the polarization vector of the external wave, at which the minimal image contrast is reached, one can get the information about the local fields inside the film. Having on hand this information and measuring the angle $\phi_{c}$ between the translation vectors of the lattice and the polarization vector of the external wave one can determine the distance between the probe and the surface.

The analysis based on eq. (25) allows one to say that formula (45) remains valid in the case of arbitrary incident angle of $s$-polarized wave as well. One has to note that in the near-field optical measurements it is more useful to work with $p$-polarized waves 36. In the case of arbitrary incidence angle of $p$-polarized wave the distribution of the field in the near zone is essentially different. This has been shown in Ref. [8] on the basis of numerical calculations. We intend to give a detailed description of the field behavior in the near zone at arbitrary incident angles in our subsequent publications.

\section{Conlcusions}

We have solved a boundary problem of the linear classical optics devoted to the investigation of the electromagnetic field behavior near the surface of a dielectric medium taking into account 
its discrete structure. The main attention has been paid to the investigation of the near-zone optical properties of dielectrics. It has been shown that at the distances from the surface less than two lattice constants the behavior of the reflected and transmitted waves is entirely different from what we have in the wave zone. The intensity distribution in the near zone allows one to determine the atomic positions at the surface.

We have shown that at the distances from the surface larger than the interatomic distance in the film the probe which measures the field does not significantly influence the field distribution in the film. In the present paper we have suggested a method of the optical control of the distance between the probe and the surface. The method is based on the analysis of the nearfield distribution at different directions of the polarization vector of the external radiation.

At first glance the system treated in our paper (ultrathin dielectric film without any substrate) seems to be unrealistic. However, it can be used for the investigation of the near-zone optical properties of the films of arbitrary thicknesses. As we have shown the near-zone optical response of a material is defined mainly by the evanescent harmonics, which play an important role only within few interatomic distances near the surface, because they decay very rapidly. This means that the "bulk" monolayers influence significantly only the wave-zone optical properties and does not change much the ratio between propagating and evanescent harmonics.

Our results can be useful not only for the development of the ultrahigh resolution near-field microscopy, but also for the investigation of the optical properties of photonic crystals in the long wavelength approximation.

The results obtained in the present paper are valid not only in the case of an ideal crystal lattice, but also when the medium has only short-range order as in the case of real surfaces. Indeed, the methods based on the Fourier transform and the Lorentz method give analogous results. At the same time only the short-range order is important for the Lorentz method and the magnitude of the dipole field is mainly determined by the atoms located at the distance from the observation point of the order of $2 \div 3$ lattice constants. The atomic distribution outside of this region does not practically influence the field.

\section{Acknowledgement}

This work has been supported in part by the Russian federal program "Integration" (grant A 0066). One of the authors (K.V.K.) is grateful to Deutsche Forschungsgemeinschaft and Alexander-von-Humboldt Stiftung for financial support. 


\section{References}

[1] Near-field optics, Ed. by D.W.Pohl and D.Courjon (Kluwer, Dordrecht, 1993).

[2] D.Barchiesi et al., Phys.Rev.E 54, 4285 (1996).

[3] R.Bachelot, P.Gleyzes and A.C.Boccara, Appl.Opt. 36, 2160 (1997).

[4] M.Xiao, JOSA A 14, 2977 (1997).

[5] M.Ashino and M.Ohtsu, Appl.Phys.Lett. 72, 1299 (1998).

[6] A.V.Zayats, Opt.Commun. 161, 156 (1999).

[7] J.Michaelis, C.Hettich, J.Mlynek, and V.Sandoghdar, Nature 405, 325 (2000).

[8] H.Miyazaki and K.Ohtaka, Phys. Rev. B. 58, 6920 (1998).

[9] I.I.Smolyaninov, W.Atia, and C.C.Davis, Phys. Rev. B 59, 2454 (1999).

[10] K.V.Krutitsky and S.V.Suhov, J.Phys.B 30, 5341 (1997).

[11] K.V.Krutitsky and S.V.Suhov, Optika i Spektroskopiya 88, 827 (2000) [Optics and Spectroscopy 88, 749-755 (2000)].

[12] G.P.M.Poppe, C.M.J.Wijers, and A.van Silfhout, Phys.Rev.B 44, 7917 (1991).

[13] G.P.M.Poppe and C.M.J.Wijers, Physica B 167, 221 (1990).

[14] O.Litzman, Opt.Acta 25, 509 (1978).

[15] O.N.Gadomsky and K.V.Krutitsky, Zh.Eksp.Teor.Fiz. 106, 936 (1994) [JETP 79, 513-523 (1994)].

[16] K.V.Krutitsky and J.Audretsch, J.Phys.B 31, 2633 (1998).

[17] A.I.Zaitsev, V.A.Malyshev, and E.D.Trifonov, Zh.Eksp.Teor.Fiz. 84, 475 (1983) [Sov.Phys.-JETP 57, 275 (1983)].

[18] V.A.Malyshev, H.Glaeske, and K.-H.Feller, Phys.Rev.A 58, 670 (1998).

[19] C.M.J.Wijers, Th.Rasing, and R.W.J.Hollering, Solid State Communications 85, 233 (1993); C.M.J.Wijers, P.L. de Boeij, C.W. van Hasselt, and Th.Rasing, Solid State Communications 93, 17 (1995).

[20] G.P.M.Poppe, R.Del Sole, and F.Manghi, Phys.Rev.B 44, 1825 (1991).

[21] W.L.Mochan and R.Barrera, Phys.Rev.Lett. 55, 1192 (1985).

[22] C.M.J.Wijers and K.M.E.Emmert, Physica Scripta 38, 435 (1988).

[23] G.P.M.Poppe, C.M.J.Wijers, and A. van Silfhout, Solid State Communications 78, 773 (1991).

[24] C.M.J.Wijers and G.P.M.Poppe, Phys.Rev. B 46, 7605 (1992). 
[25] A.Johner and P.Schaaf, Phys.Rev.B 40, 10231 (1989).

[26] A.Johner, P.Schaaf, and A.Schmitt, J.Opt.(Paris) 20, 157 (1989).

[27] A.Johner and P.Schaaf, Phys.Rev.B 42, 5516 (1990).

[28] T.Setälä, M.Kailova, and A.T.Friberg, Phys.Rev.E 59, 1200 (1999).

[29] D.V.Sivukhin, Course of general physics (Nauka, Moscow, 1985), v.4, p.432, in russian.

[30] A.Kawata, Y.Inouye, and T.Sugiura, Jpn.J.Appl.Phys., Part 2 33, L1725 (1994).

[31] S.K.Sekatskii and V.S.Letokhov, Pis'ma v Zh.Eksp.Teor.Fiz. 65, 441 (1997) [JETP Letters 65, 465 (1997)].

[32] L.Rosenfeld, Theory of electrons (North-Holland Publishing Company, Amsterdam, 1951).

[33] M.Born and E.Wolf, Principles of optics (Pergamon, NY, 1986).

[34] L.D.Landau and E.M.Lifshitz, Classical theory of fields (Addison-Wesley, NY, 1965).

[35] A.V.Ghiner and G.I.Surdutovich, Phys.Rev.E 56, 6123 (1997).

[36] O.Keller, M.Xiao, and S.Bozhevolnyi, Surf.Sci. 280, 217 (1993).

[37] O.N.Gadomsky and K.V.Krutitsky, JOSA B 13, 1679 (1996).

[38] O.N.Gadomsky and S.V.Sukhov, J.Appl.Spectr. 65, 236 (1998).

[39] A.Yariv, Optical electronics (Holt, Rinehart and Winston, NY, 1985).

[40] D.E.Aspnes and A.A.Studna, Phys.Rev.B 27, 985 (1983).

[41] O.N.Gadomsky and S.V.Sukhov, Optika i Spectroskopiya 89, 287 (2000) [Optics and Spectroscopy 89, 261 (2000)].

[42] For the description of the field near hexagonal lattice it is necessary to take into account 6 harmonics. The detailed analysis of the behavior of the near field for this case can be found in Ref. [8].

[43] C.Girard and X.Bouju, J.Opt.Soc.Am.B 9, 298 (1992).

[44] R.C.Reddick, R.J.Warmack, and T.L.Ferrell, Phys.Rev. B 39, 767 (1989). 